\title{
Kappa-distributions and Temperature Structure of the Prominence-Corona Transition Region
}

\author{
Elena Dzifčáková ${ }^{1}, \check{S ̆}$ imon Mackovjak $^{2,1}, \&$ Petr Heinzel $^{1}$ \\ ${ }^{1}$ Astronomical Institute of the Academy of Sciences of the Czech Republic, \\ Fričova 298, 25165 Ondřejov, Czech Republic, \\ email: elena.dzifcakova@asu.cas.cz, petr.heinzel@asu.cas.cz \\ ${ }^{2}$ Faculty of Mathematics Physics and Informatics, Comenius University, \\ Mlynska Dolina F2, 84248 Bratislava, Slovakia, email: mackovjak@fmph.uniba.sk
}

\begin{abstract}
The influence of the electron $\kappa$-distributions on the differential emission measure (DEM) of the prominence-corona transition region (PCTR) derived from observed line intensities has been investigated. An important consequence of the $\kappa$-distribution is formation of the emission lines in much wider temperature ranges. The implications for the formation temperature of the observed SDO/AIA band emissions are shown.
\end{abstract}

Keywords. DEM, PCTR, SUMER spectral lines, SDO/AIA

A strong gradient of temperature and density in the PCTR can form non-Maxwellian distributions with an enhanced number of particles with high energies - the $\kappa$-distribution. Therefore we have studied the influence of the $\kappa$-distributions on calculated DEM. The observed line intensities from SOHO/SUMER spectrometer listed in Parenti \& Vial (2007) as A_1 were used in our analysis. The ionization equilibria for $\kappa$-distributions were taken from Dzifčáková \& Dudík (2013) and the excitation equilibrium was calculated for atomic data corresponding to the CHIANTI 6 (Dere et al., 2009). The WithbroeSylwester method (Withbroe, 1975; Sylwester et al., 1980) was employed for the calculation of DEM. The differences between our reconstructed DEM for the Maxwellian distribution with original DEM by Parenti \& Vial (2007) (Fig. 1, top left) should be a result of different calculation methods only. The DEM's calculated for the $\kappa$-distributions are wider and flatter in comparison with DEM for the Maxwellian distribution (Fig. 1, top right; bottom left). This is mainly the result of changes in the ionization equilibrium for the $\kappa$-distributions, where the ionization peaks are wider and shifted in comparison with the Maxwellian distribution (Dzifčáková \& Dudík, 2013). For the $\kappa$-distributions, the spectral lines are formed in wider temperature ranges and the maxima of contribution to the line intensities can be substantially shifted to lower $T$, especially in transition region (Fig. 1, bottom right). These changes could affect the temperature region that is visible in SDO/AIA filters. For the AIA 171 and 193 filters, and Fe X-XII lines (Fig. 2), the maxima of contributions to the line intensity have similar positions for both Maxwellian and $\kappa$-distributions. However, the Fe IX and Fe VIII lines show enhanced low-temperature contributions and the contributions from O V and O VI lines, formed at even lower temperatures, are significantly widened. We can conclude that the $\kappa$-distributions allow to see wider temerature range in AIA filters than the Maxwellian one. 

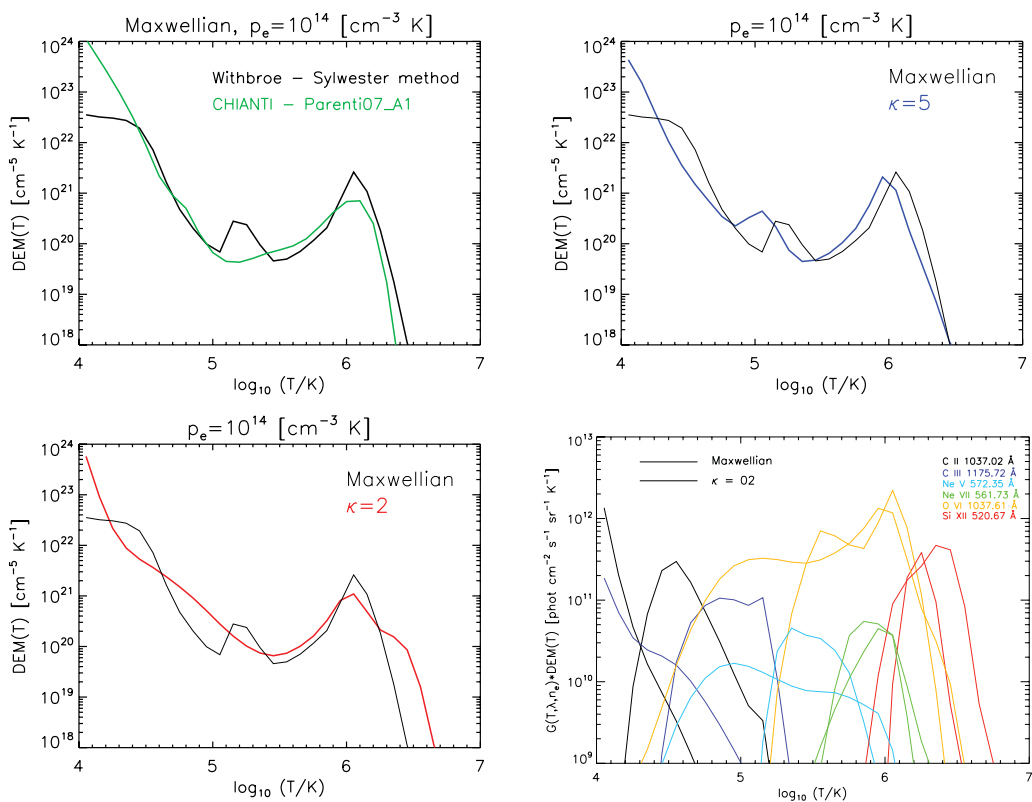

Figure 1. Top left: The PCTR DEM for the Maxwellian distribution by Parenti \& Vial (2007) (green line) and by Withbroe-Sylwester method (black line). Top right: DEM for the $\kappa$-distribution with $\kappa=5$ (blue line). Bottom left: DEM for the $\kappa$-distribution with $\kappa=2$ (red line). Bottom right: $G\left(T, \lambda, n_{e}\right) * \mathrm{DEM}$ for the Maxwellian (full lines) and for the $\kappa$-distributions with $\kappa=2$ (dot-dot-dot dashed lines) for six lines formed in different temperature ranges.
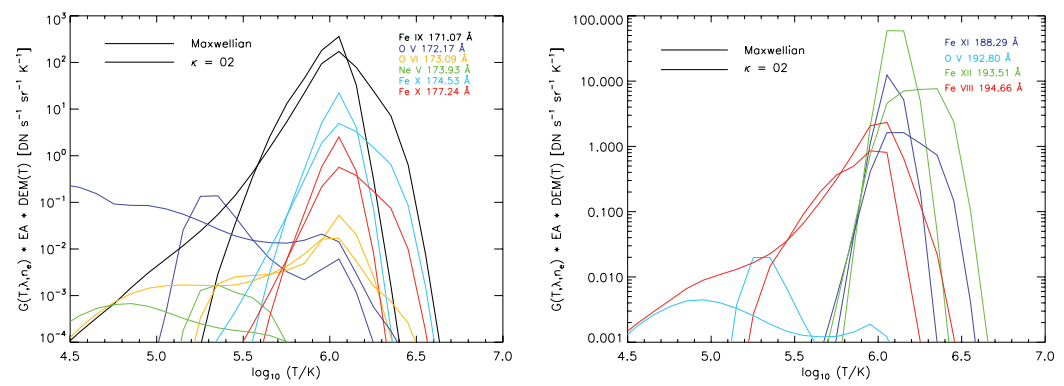

Figure 2. $G\left(T, \lambda, n_{e}\right) *$ DEM multiplied by the effective area (EA) of 171 (left) and 193 band (right) for the Maxwellian (full lines) and $\kappa$-distribution with $\kappa=2$ (dot-dot-dot dashed lines).

\section{Acknowledgements}

This work has been supported by Grant No. 209/12/1652 of the Grant Agency of the Czech Republic, grant No. 1/0240/11 of Scientific Grant Agency VEGA Slovakia, and the bilateral project APVV CZ-SK-0153-11 (7AMB12SK154) involving the Slovak Research and Development Agency and the Ministry of Education of the Czech Republic.

\section{References}

Dzifčáková, E. \& Dudík J., 2013, ApJS, 206, 6

Dere, K. P., Landi, E., Young, P. R., Del Zanna, G., Landini, M., \& Mason, H. E., 2009, A\& A, 498, 915

Parenti, S. \& Vial J. C., 2007, A\&AA, 469, 1109

Sylwester, J., Schrijver, J., \& Mewe, R., 1980, SoPh, 67, 285

Withbroe, G. L., 1975, SoPh, 45, 301 